\title{
LEVANTAMENTO QUALIQUANTITATIVO DA ARBORIZAÇÃO URBANA DO BAIRRO FERRAROPÓLIS NA CIDADE DE GARÇA-SP
}

Raphael Layola Nunes $^{1}$; Caio Vinicius Ferreira Marmontel ${ }^{3}$; João Paulo Rodrigues ${ }^{4}$; Augusto Gabriel Claro

$$
\mathrm{Melo}^{5}
$$

\section{RESUMO}

As análises da arborização urbana auxiliam para possíveis melhorias no planejamento e gestão das áreas verdes urbanas. O objetivo foi realizar o levantamento qualiquantitativo do bairro Ferraropólis, município de Garça, SP, servindo como base para definição de medidas de possíveis recomendações de manejo a serem aplicadas. $\mathrm{O}$ método adotado para o inventário foi censo total, identificando cada indivíduo além da avaliação dos parâmetros de condições fitossanitárias, qualidade de poda, interferência na rede elétrica e calçada. Foram amostrados 1.516 indivíduos, pertencentes a 55 espécies, 49 gêneros e 25 famílias. A espécie com maior abundância foi a Lagerstroemia indica, com 268 indivíduos e a família Fabaceae apresentou maior riqueza com 20 espécies. Das árvores catalogadas, 561 estão com sua condição boa, 189 necessitam de controle fitossanitário e 766 necessitam de podas leves ou de limpeza e a interferência na fiação e calçadas foi baixa devido as constantes podas. As espécies ocorrem em uma distribuição heterogênea dos indivíduos, ocorrendo devido ao plantio aleatório de órgãos municipais ou até mesmo da população, além do plantio de espécies exóticas por ser atrativa em sua estética, ao invés de inserir espécies nativas da região.

Palavras-chave: Área verde urbana; árvores; Espécies nativas; Planejamento urbano; Recomendações de manejo.

\section{SURVEY QUALI-QUANTITATIVE OF URBAN FORESTRY IN THE NEIGHBORHOOD FERRAROPOLIS IN THE CITY OF GARÇA-SP}

\begin{abstract}
The analyzes of urban forestry auxiliary for possible improvements in the planning and management or urban green areas. The objective was to survey the qualitative and quantitative Ferraropolis neighborhood, in the city of Garça, SP, serving as basis for defining measures of possible management recommendations to be implemented. The method adopted for the inventory was complete census, identify each individual and by evaluating the parameters of phytosanitary conditions, quality of pruning, interference with grid and sidewalk. We sampled 1,516 individuals belonging to 55 species, 49 genres and 25 families. The most abundant species was the Lagerstroemia indica, with 268 individuals and family Fabaceae showed grater richness with 20 species. Cataloged trees, 561 are in good condition, require phytosanitary control 189 and 766 require light pruning or cleaning and the interference in wiring and sidewalks was low from constant pruning. The species occurs in a heterogeneous distribution of individuals, occurring due random planting of municipal bodies or even the population, and the planting of exotic species to be attractive it's a esthetic instead of region native species.
\end{abstract}

Key-words: Urban green areas; Trees; Native species; Urban planning; Management of recommendation.

\footnotetext{
${ }^{1}$ Eng Florestal da Fac. Agronomia e Engenharia Florestal/Assoc. Cultural e Educacional de Garça - SP. e-mail: raphaellayola@hotmail.com ${ }^{2}$ recebido em 24.05.2012 e aceito para publicação em 15.03.2013

${ }^{3}$ Eng Florestal, Mestrando em Ciências Florestais - Universidade Estadual Paulista - Fac. Ciências Agronômicas, Botucatu - Departamento de Ciências Florestais/Laboratório de Hidrologia Florestal, Botucatu, SP. e-mail: marmontel.cvf@fca.unesp.br

${ }^{4}$ Eng Florestal da Fac Agronomia e Engenharia Florestal/Assoc. Cultural e Educac. de Garça - SP. E-mail: rodriguesflorestal@hotmail.com

${ }^{5}$ Eng Florestal, Mestre em Eng. Florestal, Coord. e Docente do curso de Engenharia Florestal - Fac. Agronomia e Eng Florestal/Assoc.

Cultural e Educacional de Garça - SP. E-mail: augustogabriel.ef@hotmail.com
} 
A arborização urbana vem merecendo uma atenção cada vez maior, devido aos benefícios e os problemas apresentados em função da presença da árvore inserida na cidade, e com isso pesquisas aprofundadas têm sido cada vez mais valorizados, através da execução de monitoramento periódico que permita avaliação de aspectos qualiquantitativos a partir das características das árvores (DANTAS; SOUZA, 2004). Apesar de todo destaque que se dá sobre a importância de estabelecer um planejamento na arborização das cidades, pelos vários tipos de benefícios que essa vegetação proporciona não se obtiveram muitos avanços, pois poucas cidades brasileiras possuem um inventário e um plano de manejo para suas árvores (TAKAHASHI, 1994).

Um aspecto importante da arborização de ruas se refere ao seu planejamento adequado, de forma que, ao se desenvolver, a árvore não entre em conflito com outras estruturas urbanas, como fiação aérea, tubulação subterrânea, o tráfego e outros (VILLARINHO; MACEDO; TOMIAZZI, 2005). Um mal planejamento é um procedimento muito comum, causando vários prejuízos, como rompimento de fios de alta-tensão, interrupções no fornecimento de energia elétrica, entupimento de redes de esgoto e calhas, rachaduras em calçadas, obstáculos para circulação e acidentes envolvendo pedestres, veículos ou edificações (TAKAHASHI, 1992).

Como o objetivo de buscar uma maior heterogeneidade é relevante a elaboração de um plano de arborização urbana, obtendo um diagnóstico preciso das vias arborizadas, de acordo com a legislação pertinente, e que atenda aos anseios da população e ao equilíbrio ambiental (MIRANDA; CARVALHO, 2009).

Através da realização do inventário torna-se possível analisar as condições ideais para um bom desenvolvimento da arborização urbana, buscando assim informações necessárias para desenvolver um plano de manejo adequado, permitindo conhecer condições da arborização em termos de adaptabilidade e problemas relacionados as espécies (PIVETTA; SILVA FILHO, 2002). Com os resultados obtidos com esses estudos torna-se possível auxiliar o planejamento e o manejo da arborização, fornecendo informações sobre necessidades de poda, tratamentos fitossanitários, intervenções silviculturais (SILVA; GONÇALVES; PAIVA 2007).

O trabalho teve como objetivo realizar o levantamento qualiquantitativo da arborização do bairro Ferraropólis na cidade de Garça, servindo como base para definição de medidas de possíveis recomendações de manejo a serem aplicadas.

\section{MATERIAIS E MÉTODOS}

\section{Caracterização da área de estudo}

O estudo foi realizado no bairro Ferraropólis, município de Garça, região centro-oeste do estado de São Paulo. Está localizado nas coordenadas: $22^{\circ}$ 12' Sul e $49^{\circ} 39^{\prime}$ Oeste a 690 metros do nível do mar (Figura 1). O bairro está situado no setor central do município, caracterizado como 
residencial e comercial e apresenta 80 quarteirões, sendo o mais antigo da cidade, fundado em 1938. O clima do município é subtropical, com temperatura média anual de $28,5^{\circ} \mathrm{C}$, precipitação média anual de $1.274,4 \mathrm{~mm}$ e o tipo de solo é Podzólico Vermelho Amarelo (EMBRAPA, 2006).

\section{Levantamento qualiquantitativo da arborização}

O método adotado para o levantamento quantitativo foi o censo total - todos os indivíduos foram amostrados durante os meses de outubro a dezembro de 2011. A cada contato com os indivíduos era identificada a espécie utilizando o sistema de classificação APG (2009), além da definição de cada indivíduo se era nativa ou exótica, denominação do hábito de cada indivíduo, podendo ser de porte arbóreo, arbusto ou palmeira.
Para o levantamento qualitativo foram utilizados quatro parâmetros:

- condições fitossanitárias: 'boa', quando o indivíduo apresentava-se sem sinais aparentes de ataques de pragas, doenças ou injúrias mecânicas; 'ruim', quando apresentaram sinais de ataques.

- Qualidade de poda: 'boa', copa bem estruturada, não havendo necessidade de poda; 'necessitando', interferindo na fiação, automóveis, pedestres; 'ruim', retirada quantidade excessiva de ramos, denominada poda drástica.

- Interferência na rede elétrica: 'não', ramos da copa não estavam em contato com os cabos da energia elétrica ou telefônica; 'sim', quando os ramos da copa estavam em contato com os cabos.

- Interferência na calçada: 'não', raízes não expostas e sem danos nas calçadas; 'sim', raízes expostas com danos nas calçadas.

Figura 1. Vista aérea (destaque de vermelho) do bairro Ferrapólis, na cidade de Garça, SP

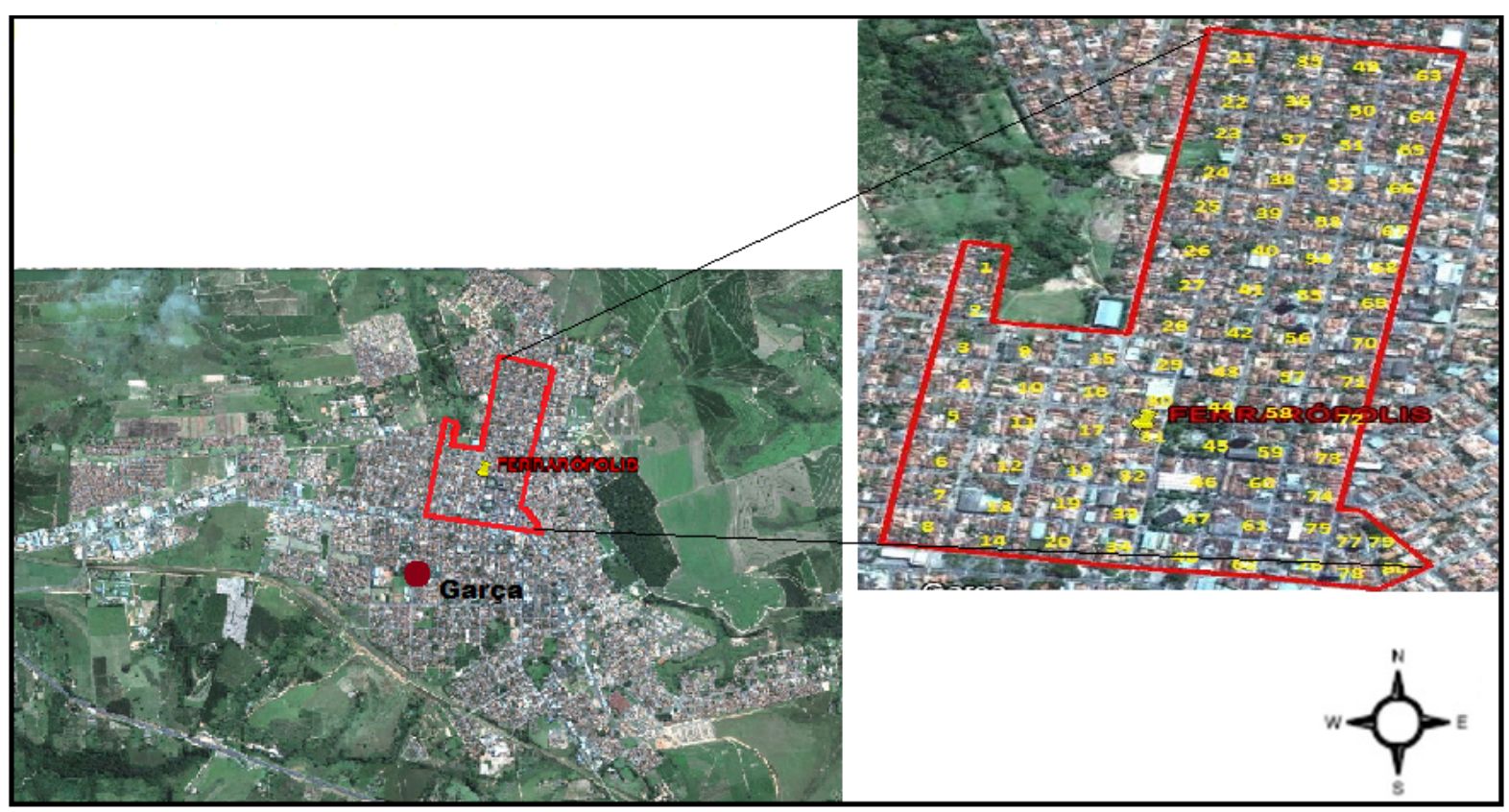

Fonte: Adaptado do Google Earth / Source: Adapted of Google Earth. 


\section{Levantamento quantitativo}

Foram amostrados 1.516 indivíduos vivos, sendo $50,1 \%$ árvores, $49,2 \%$ arbustos e $0,7 \%$ palmeiras, distribuídas em 55 espécies, 49 gêneros e 25 famílias (Tabela 1). No estudo de Palharini et al. (2007, 2010) nos bairros Araceli e Labienopólis
(Garça) encontraram variações no números de indivíduos $(776,1609)$ e homogeneidade em número de espécies $(61,61)$. Essa diferença na quantidade de indivíduos comparados com outros bairros da cidade pode ser resultado da dimensão territorial, ou seja, maior área amostral.

Tabela 1. Lista das espécies encontradas na arborização urbana do bairro Ferraropólis, Garça, SP

\begin{tabular}{|c|c|c|c|c|}
\hline Família & Espécie & Origem & Hábito & F (\%) \\
\hline \multirow{5}{*}{$\begin{array}{l}\text { Aceraceae } \\
\text { Anacardiaceae }\end{array}$} & Aceae palmatum Thunb. & $\mathrm{E}$ & Arv & 0,07 \\
\hline & Schinnus molle L. & $\mathrm{N}$ & Arv & 3,36 \\
\hline & Schinnus terebintifolius Raddi. & $\mathrm{N}$ & Arv & 0,33 \\
\hline & Mangifera indica L. & $\mathrm{E}$ & Arv & 0,26 \\
\hline & Spondias purpuera L. & $\mathrm{E}$ & Arv & 0,07 \\
\hline \multirow[t]{2}{*}{ Apocyanaceae } & Nerium oleander L. & $\mathrm{E}$ & Arb & 8,58 \\
\hline & Thevetia thevetioides (Kunth) K Schum & $\mathrm{E}$ & Arv & 0,13 \\
\hline \multirow[t]{2}{*}{ Arecaceae } & Dypsis lutescens (H.Wendl.) Beentje \& J.Dransf. & $\mathrm{N}$ & Palm & 0,07 \\
\hline & Roystonea regia (Kunth) O. F. Cook & $\mathrm{E}$ & Palm & 0,66 \\
\hline \multirow[t]{6}{*}{ Bignoniaceae } & Tecoma stans (L.) Juss. Ex Kunth & $\mathrm{E}$ & Arb & 1,58 \\
\hline & Handroanthus chrysotrichus (Mart. ex A.DC.) Mattos & $\mathrm{N}$ & Arv & 1,39 \\
\hline & Handroanthus albus (Cham.) Mattos & $\mathrm{N}$ & Arv & 0,92 \\
\hline & Jacaranda mimosifolia D. Don & $\mathrm{E}$ & Arv & 0,86 \\
\hline & Handroanthus impetiginosus (Mart. ex DC.) Mattos & $\mathrm{N}$ & Arv & 0,46 \\
\hline & Spathodea nilotica Seem & $\mathrm{E}$ & Arv & 0,13 \\
\hline Casauarenaceae & Casuaria torulosa Dryand \& Ait. & $\mathrm{E}$ & Arv & 0,13 \\
\hline Chrysolanaceae & Licania tomentosa (Benth.) Fritsch & $\mathrm{N}$ & Arv & 10,3 \\
\hline Compretaceae & Terminalia catappa L. & $\mathrm{E}$ & Arv & 0,73 \\
\hline \multirow{10}{*}{$\begin{array}{l}\text { Euphorbiaceae } \\
\text { Fabaceae }\end{array}$} & Euphorbia leucocephala Lotsy & $\mathrm{E}$ & Arb & 0,07 \\
\hline & Caesalpinia pluviosa Benth. & $\mathrm{N}$ & Arv & 4,29 \\
\hline & Caesalpinia pulcherrima (L.) Sw. & $\mathrm{E}$ & Arb & 3,3 \\
\hline & Poecilanthe parviflora Benth. & $\mathrm{N}$ & Arv & 2,51 \\
\hline & Bauhinia purpurea L. & $\mathrm{E}$ & Arv & 2,31 \\
\hline & Senna didymobotrya (Fresen.) H. Irwin \& Barnaby & $\mathrm{E}$ & Arv & 0,92 \\
\hline & Tipuana tipu (Benth.) Kuntze & $\mathrm{E}$ & Arv & 0,73 \\
\hline & Senna bicapsularis (L.) Roxb. & $\mathrm{N}$ & Arb & 0,33 \\
\hline & Pterocarpus violaceus Vogel & $\mathrm{N}$ & Arv & 0,07 \\
\hline & Bacharis dracunculifolia DC. & $\mathrm{N}$ & Arb & 0,07 \\
\hline
\end{tabular}


Erytrina indica Lam. var. picta Hort.

\begin{tabular}{ccc} 
E & Arv & 0,07 \\
E & Arv & 0,07 \\
N & Arv & 0,4 \\
E & Arv & 0,07 \\
E & Arb & 17,7 \\
E & Arv & 3,83 \\
E & Arb & 0,26 \\
N & Arv & 10,1 \\
E & Arb & 0,46 \\
N & Arv & 0,07 \\
N & Arb & 1,65 \\
E & Arv & 0,66 \\
E & Arb & 0,07 \\
E & Arv & 0,4 \\
N & Arb & 0,13 \\
E & Arv & 0,13 \\
E & Arv & 0,99 \\
E & Arb & 1,52 \\
E & Arv & 0,73 \\
E & Arb & 0,07 \\
E & Arb & 13,3 \\
N & Arv & 0,99 \\
E & Arb & 0,2 \\
E & Arv & 0,2 \\
E & Arv & 0,13 \\
E & Arv & 0,07 \\
E & Arv & 1,25 \\
\hline & & 100 \\
\hline
\end{tabular}

Lauraceae

Liliaceae

Lytharaceae

Malpighaceae

Malvaceae

Melastomataceae

Moraceae

Myrtaceae

Olecaceae

Proteaceae

Rosaceae

Rutaceae

Salicaceae

Muntingiaceae

Verbenaceae
Leucena leucocephala (Lam.) R. de Wit

Nectandra megapotamica (Spreng.) Mez

Dracaena fragrans (L.) Ker-Gawl.

Lagerstroemia indica $\mathrm{L}$.

Lagerstroemia speciosa Pers.

Malpighia glabra L.

Pachira aquatica (L.) Jacq.

Hibiscus rosa sinensis L.

Bombacopsis glabra (Pasq.) A. Robyns

Tibouchina granulosa (Desr.) Cogn.

Ficus benjamina $L$.

Morus nigra L.

Callistemon viminalis (Sol. Ex Gaertn.) G. Don ex Loud.

Psidium guajava $\mathrm{L}$.

Syzygium cumini (L.) Skeels

Ligustrum lucidum W. T. Aiton

Grevillea banksii R. Br.

Grevillea robusta A. Cunn. Ex. R. Br.

Prunus campanulata Maxim.

Murraya paniculata (L.) Jacq.

Dicttyoloma vandellianum A. Juss.

Citrus limonia L. Osbeck

Populus alba L.

Salix alba L.

Muntingia calabura $\mathrm{L}$.

Calicarpa reevesii Wall. ex Walp

\section{$\sum$}

Em que: $\mathrm{N}$ = nativa, $\mathrm{E}=$ exótica, Arv = árvore, $\mathrm{Arb}=$ arbusto, Palm = palmeira, $\mathrm{F}(\%)=$ frequência em porcentagem.

A família mais representativa foi a Fabaceae, com 11 espécies distintas (20\%) e 222 indivíduos (14,6\%). A Fabaceae é considerada a família mais rica e abundante nas florestas da América do Sul (HUECK, 1972). A obtenção de mudas por propagação sexuada com facilidade, crescimento rápido em meio urbano além do potencial de estética das espécies justificam serem inseridas em proporções elevadas quando comparada com as outras (JACINTO, 2001). O destaque da família já era esperado, uma vez que essa família é característica de florestas semidecíduas, característica da região.

A espécie com maior número de indivíduos foi a Lagerstroemia indica (resedá) 268 (17,7\%), seguida da Murraya paniculata (falsa-murta) 201 (13,3\%), Licania tomemtosa (oiti) 156 (10,3\%), Pachira aquatica (monguba) 153 (10,1\%) e Nerium oleander (espirradeira) 130 (8,6\%), sendo essas espécies responsáveis por $60 \%$ do total de indivíduos presentes no bairro. Silva (2000) afirma serem comum na arborização urbana, poucas 
espécies representarem a maior parte da população, mesmo não sendo uma situação desejável.

O resedá e a espirradeira são espécies exóticas e ornamentais, principalmente pela intensa floração, adequada para arborização de ruas, sendo o resedá a principal espécie plantada nas cidades do sul do Brasil (LORENZI et al., 2003). A monguba possui frutos grandes e pesados que possam causar alguns acidentes, além do vigor nas raízes superficiais, porém proporciona uma melhoria no microclima (RIZZO, 1975). O oiti tem sido largamente utilizado na arborização devido a sua copa frondosa e perene (TUDINI, 2006), mas seu uso em grande escala deve ser evitado por se tratar de grande porte e o aparecimento de ferrugem causada por Phakopsora tomentosae, que ocasiona a morte das plantas (FERREIRA; GASPAROTO; LIMA, 2001).

A Lagerstroemia indica está fora do padrão proposto por Grey e Deneke (1978), que permite uma frequência de 10 até $15 \%$ de uma espécie, com porcentagens mais elevadas aumentam o risco de ocorrência de pragas e doenças. É comum na arborização urbana ocorrer poucas espécies representando a maioria da população, mesmo não sendo uma situação desejável, que por razões estéticas ou fitossanitárias (SILVA, 2000).

Considerando a origem das 55 espécies encontradas, verificou-se que $37(67,3 \%)$ são exóticas e 18 (32,7\%) nativas, contabilizando em indivíduos foram $949(62,6 \%)$ exóticas e 567 $(37,4 \%)$. No bairro Labienopólis (Garça, SP),
68,8\% das espécies foram exóticas e 31,1\% nativas (Palharini et al., 2010). É comum o plantio de espécies exóticas em projetos de arborização urbana, por falta de um prévio conhecimento, mas não devem ser descartadas pois acabam trazendo alguns benefícios, tais como melhoria do clima e a estética, entretanto as espécies nativas regionais devem ser priorizadas por seu valor sociocultural e sua variabilidade genética (SILVA et al. 2002). O uso de espécies nativas diminui os riscos de desequilíbrio ambiental causados pela influência de espécies exóticas na biodiversidade de ecossistemas urbanos.

\section{Levantamento qualitativo}

As condições fitossanitárias dos indivíduos apresentaram 1.327 (87,5\%) com condições sadias e vigor, 189 (12,5\%), ruins, ou seja, com injúrias mecânicas, pragas e doenças aparentes comprometendo sua sobrevivência (Tabela 2). Esse valor foi inferior se comparados aos bairros Araceli (97\%) boa e Labienopólis (98\%) nos estudos de Palharini et al. (2007, 2010); valor superior foi encontrado no estudo de Sbrissa et al. (2011) no bairro J. K. Williams onde obteve $77,8 \%$ com condições boas de fitossanidade. Milano (1994) menciona que para se alcançar o desenvolvimento satisfatório qualidade das árvores é necessário, também o planejamento prévio de práticas de manutenção, como monitoramento, irrigação, adubação, poda e controle fitossanitário.

Tabela 2. Parâmetros qualitativos avaliados das espécies arbóreas e arbustivas que compõem a arborização do bairro Ferraropólis, na cidade de Garça, SP

\begin{tabular}{cccccccccc}
\hline & \multicolumn{2}{c}{ Fitossanidade } & \multicolumn{3}{c}{ Qual. de poda } & \multicolumn{2}{c}{ Interf. na fiação } & \multicolumn{2}{c}{ Interf. na calçada } \\
& Bom & Ruim & Bom & Necessitando & Ruim & Sim & Não & Sim & Não \\
\hline NI & 1327 & 189 & 1070 & 240 & 206 & 215 & 1301 & 562 & 954 \\
& & & & & & & & & Raphael Layola Nunes et al..
\end{tabular}




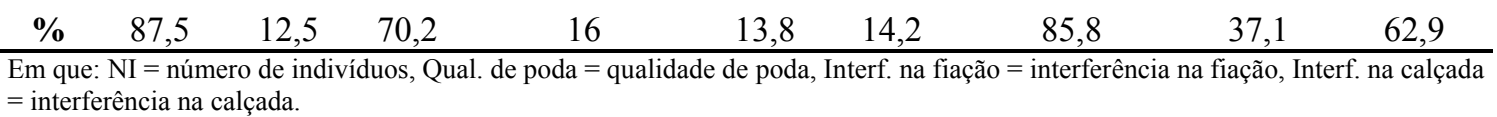

As espécies que apresentaram fitossanidade ruim na maioria das vezes são árvores de grande porte, podendo ser motivo da poda drástica ou falta de conhecimento das técnicas para a realização de podas, sendo assim, acabam danificando o indivíduo além de favorecer a entrada de patógenos, produzindo o apodrecimento do lenho e causando até a morte da planta.

$\mathrm{Na}$ análise da qualidade de poda, verificou-se que 1.070 indivíduos $(70,2 \%)$ estão boas, 240 (16\%) estão necessitando de podas, por motivos de interferência na rede elétrica além da dificuldade da passagem de pedestres e veículos; $206(13,8 \%)$ apresentaram poda drástica, No estudo de Palharini et al. (2010) encontrou valor inferior ao do estudo $(3,7 \%)$ de podas drástica no bairro Labienopólis. Este valor superior pode ser explicado pelo bairro Ferraropólis ser uma área comercial, onde as podas drásticas são aplicadas para principalmente não danificar pedestres e automóveis, além da fiação, isso é o motivo de ser inserida uma espécie inadequada para o local por falta de conhecimento do órgão público municipal ou dos próprios moradores, principalmente onde possui a fiação que acaba tendo que realizar contínuas podas de condução, comprometendo também a forma típica da árvore, tirando a sua estética e exuberância da espécie. As melhores copas se encontram nas vias onde não há presença da rede elétrica, sendo resultados de poucas ações de podas, não prejudicando o indivíduo e sua arquitetura.

As podas drásticas são irrelevantes, pois após sua execução ocorre uma super brotação nas proximidades dos cortes e novos ramos tendem a uma posição ascendente, com isso ocorre o aumento da seiva favorecendo o desenvolvimento em altura e problemas novos irão existir (BALLENSIEFER; WIECHESTECK, 1985). O ideal é que sejam realizadas podas periódicas de manutenção e com especialistas na realização dessas atividades.

Em relação a interferência da copa das árvores na fiação elétrica, 1.301 indivíduos $(85,8 \%$ do total) não estão interferindo e 215 (14,2\%) apresentaram interferência. Esse valor de interferência foi menor ao encontrado por Palharini et al. (2007, 2010). Essa alta proporção de indivíduos interferindo na fiação provavelmente ocorreu devido as diversas manutenções das espécies arbóreas de porte alto além da presença de arbusto com porte baixo que não alcançam a fiação. Lorenzi et al. (2003) afirma que nas calçadas de ruas sob fiação elétrica apenas espécies com menos de 8 metros de altura quando adultas, podem ser plantadas na arborização.

.Uma sugestão seria a implantação da rede de energia elétrica nas calçadas oeste e norte, e sob elas árvores de pequeno porte e nas calçadas leste e sul, árvores de porte médio. No caso de árvores com porte inadequado para plantio sob fiação, cujas copas estão em contato com a rede aérea, uma opção é implantar soluções de redes isoladas, protegidas ou compactas, que permitam melhor convivência com a arborização existente.

Aos danos ocasionados pelo sistema radicular, constatou-se que 954 indivíduos (63\%) não apresentaram danos nas calçadas do bairro e 562 (37\%) apresentaram danos. Valor superior foi encontrado na interferência das calçadas no bairro Araceli com $45 \%$ dos indivíduos (PALHARINI et al., 2007). A falta de área livre provoca um mau 
desenvolvimento das plantas, e a utilização de espécies com sistema radicular pouco profundo numa área pequena, pode comprometer a calçada, devido a pressão exercida pelas raízes, sendo recomendando uma área livre superior a $1 \mathrm{~m}^{2}$. (SANTOS E TEIXEIRA, 2001).

As espécies que apresentaram maiores danos as calçadas foram: Ficus benjamina (figueira), Jacaranda mimosifolia (jacarandá), Caesalpinia pluviosa (sibipiruna), Syzygium cumini (jambolão), Leucena leucocephala (leucena), Licania tomentosa (oiti) e Pachira aquatica (monguba). Paula e Melo (2010) citam as espécies de figueira, monguba, jambolão e oiti que causaram maiores danos nas calçadas da cidade de Planalto, SP.
As árvores que apresentam maiores danos as calçadas são as que possuem sistema radicular superficial, com isso torna-se fundamental conhecer as características das árvores antes de inserir no ambiente urbano (ELETROPAULO, 1995).

\section{Possíveis recomendações de manejo}

De acordo com os parâmetros qualitativos, foi verificado que $37 \%$ dos indivíduos não necessitam de manejo, apresentando qualidade satisfatória no local urbano inserido.

Tabela 3. Porcentagem dos tipos de recomendações de manejo para os indivíduos que compõem a arborização do bairro Ferraropólis, na cidade de Garça, SP

\begin{tabular}{ccc}
\hline Recomendações & NI & F (\%) \\
\hline Podas leves & 680 & 45 \\
Não necessita & 561 & 37 \\
Controle fitossanitário & 189 & 12,5 \\
Podas de limpeza & 86 & 5,7 \\
\hline$\sum$ & 1516 & 100 \\
\hline \multicolumn{3}{c}{ Em que: NI = número de indivíduos, F (\%) } \\
= frequência em porcentagem.
\end{tabular}

As podas leves são recomendadas para $45 \%$ dos indivíduos, afim de reduzir os conflitos das árvores com a rede aérea, com vistas a diminuir a brotação de ramos e conseqüentemente a intensidade de podas posteriores e a poda de limpeza é necessária em 5,7\% dos indivíduos. Para ser executadas podas é necessário ter uma grande experiência e pertencer a equipe da Prefeitura Municipal ou pela Secretaria de Agricultura e Meio Ambiente (SAMA) além de usar equipamentos de segurança e proteção individual. O controle de pragas e doenças das árvores deve ser realizado em 12,5\% dos indivíduos através de técnicos habilitados, avaliando cada indivíduo e emitindo um diagnóstico com pretensão de indicar alguma solução para o problema, entretanto, se estiver totalmente infestada pode ocorrer a sua substituição, pois não é recomendado o controle químico de pragas e doenças no ambiente urbano. 
As espécies ocorrem em uma distribuição heterogênea dos indivíduos, existindo poucas espécies com muitos indivíduos e muitas espécies com poucos indivíduos, onde deveria ter uma homogeneidade no ambiente urbano. Isso acaba ocorrendo devido ao plantio aleatório de órgãos municipais ou até mesmo da população, além do plantio de espécies exóticas por ser atrativa em sua estética, ao invés de inserir espécies nativas da região.
Uma forma de conscientizar a população de plantar as espécies adequadas é a extensão da educação ambiental, sendo uma forma de conscientizar a comunidade sobre assuntos em geral da arborização urbana além do SAMA e a prefeitura realizarem manutenção e fiscalização eficazes, uma boa gestão e política pública além de seguirem corretamente toda a legislação apropriado para a arborização urbana.

\section{AGRADECIMENTOS}

Aos amigos-irmãos das repúblicas Raiz, Jardim Jah, Tradicional e Há Mato na ajuda da coleta de dados intensa, redação científica do trabalho, Prefeitura e o SAMA da cidade de Garça, meus sinceros agradecimentos.

\section{REFERÊNCIAS BIBLIOGRÁFICAS}

APG. An update of the Angiosperm Phylogeny Group classification for the orders and families of flowering plants: APG III. Botanical Journal of the Linnean Society, London, v. 16, p. 105-121, out. 2009.

BALENSIEFER, M.; WIECHETECK, M. Arborização de cidades. Curitiba: Instituto de Cartografia e Florestas. 1987.

DANTAS, I. C.; SOUZA, C. M. C. Arborização Urbana na cidade de Campina Grande - PB: Inventário e suas espécies. Revista de Biologia e Ciências da Terra, Campina Grande v. 4, n.2, p. 18, jul./dez. 2004.

ELETROPAULO. Guia de planejamento e manejo da arborização urbana. São Paulo: Eletropaulo; CESP; CPFL, 1995. $38 \mathrm{p}$.

EMBRAPA. Centro Nacional e Pesquisa em Solos. Sistema Brasileiro de Classificação de Solos. Brasília: Embrapa-SPI. Rio de Janeiro: Embrapa-Solos. 2006, 306 p.

FERREIRA, F. A., GASPAROTTO, L.; LIMA, M. I. P. M. Uma ferrugem, causada por Phakopsora tomentosae em oiti, em Manaus. Fitopatologia brasileira, Brasília, v. 26, n. 2, p. 206-208, jun. 2001.

GREY, G. W.; DENEKE, F. J. Urban forestry. New York: John Wiley, 1978.

HUECK, K. As florestas da América do Sul. Ecologia, composição e importância econômica. São Paulo: Universidade de Brasília; Polígono, 1972. 465p.

JACINTO, J. M. M. Análise silvicultural urbana de seis espécies florestais utilizadas na arborização de Brasília. 2001. 65f. Dissertação (Mestrado em Ciências Florestais) - Universidade de Brasília, Brasília. 2001.

LORENZI, H.; SOUZA, H. M.; TORRES, M. A. V.; BACHER, L. B. Árvores Exóticas no Brasil: madeireiras, ornamentais e aromáticas. Nova Odessa: Instituto Plantarum, 2003, 368 p.

LEVANTAMENTO QUALIQUANTITATIVO DA ARBORIZAÇÃO... 
MILANO, M. S. Métodos de amostragem para avaliação de ruas. In: CONGRESSO BRASILEIRO SOBRE ARBORIZAÇÃO URBANA, 2., São Luiz. Anais... São Luiz, MA: SBAU, 1994. p.163-168.

MIRANDA, T. O.; CARVALHO S. M. Levantamento quantitativo e qualitativo de indivíduos arbóreos presentes nas vias do bairro da ronda em Ponta Grossa - PR. Revista da Sociedade Brasileira de Arborização Urbana, Piracicaba, v.4, n.3, p.143-157, jul./set. 2009.

PALHARINI, L. A. S.; MARMONTEL, C. V. F.; SANTOS, L. J. TERRA, P. M. M. MARINO JÚNIOR, E. Levantamento da arborização urbana do bairro Labienopólis, munícipio de Garça, SP. In: SIMPÓSIO DE CIÊNCIAS APLICADAS DA FAEF, 13., Garça, SP. Anais... Garça: FAEF, v.3, 2010, p. 211-217 (CD-ROM).

PALHARINI, L. A. S.; PEREIRA, A. P.; LIMA, O. Z.; DELGADO, L. G. M.; ALHO, A.; SACCÁ, J. P.; GORENSTEIN, M. R. Estudo da arborização urbana do bairro Araceli, Município de Garça, SP. In: SIMPÓSIO DE CIÊNCIAS APLICADAS DA FAEF, 10., Garça, SP. Anais... Garça: FAEF, v.2. 2007. p. 213-219. (CD-ROM).

PAULA, D. S.; MELO, A. G. C. Levantamento quali-quantitativo da arborização urbana do município de Planalto, SP. Revista Científica Eletrônica de Engenharia Florestal, Garça, v. 16, n.1, p.64-81, ago. 2010.

PIVETTA, K. F. L.; SILVA FILHO, D. F. Arborização urbana. Jaboticabal: UNESP/FCAV/FUNEP, 2002. 74p. (Boletim Acadêmico. Série Arborização Urbana).

RIZZO, J. A. Relação da arborização de áreas verdes com densidade populacional de Goiânia. In: CONGRESO IBEROAMERICANO DEL MÉDIO AMBIENTE, 3., Madrid, España. Anais... Madrid, España: C.E.M.A. 1975, p. 17491772 .

SANTOS, N. R. Z.; TEIXEIRA, I. F. Arborização de vias públicas: ambiente x vegetação.

Porto Alegre: Instituto Souza Cruz, 2001. 135 p.

SBRISSA, F. C.; SUBIRA, V. L.; FUKUMOTO, H. K.; MELO, A. G. C.; MARMONTEL, C. V. F. Levantamento da arborização urbana de um trecho do bairro 'J. K. Williams' - Garça, SP. In: Simpósio de Ciências Aplicadas da FAEF, 14. , 2011, Garça. Anais... Garça : FAEF, v. 3, 2011. p. 211-217.

SILVA, A. G. Arborização urbana em cidades de pequeno porte: avaliação quantitativa e qualitativa. 2000. $150 \mathrm{f}$. Dissertação (Mestrado em Ciências Florestais) - Universidade Federal de Viçosa, Viçosa, MG. 2000.

SILVA, A. G., GONÇAlVES, W.; PAIVA, H. N. Avaliando a arborização urbana. Viçosa, MG: Aprenda Fácil, 2007. $346 p$.

SILVA, E. M.; SILVA, A. M.; MELO, P. H.; BORGES, S. S.; LIMA, S. C. Estudo da arborização urbana do bairro Mansour na cidade de Uberlândia-MG. Caminhos de Geografia, Uberlândia, v. 3, n.5, p. 73-83, fev. 2002.

TAKAHASHI, L. Y. Arborização urbana: Inventário. In: CONGRESSO BRASILEIRO DE ARBORIZAÇÃO URBANA, 2. ENCONTRO NACIONAL SOBRE ARBORIZAÇÃO URBANA, 5., São Luis, MA. Anais... São Luís: Prefeitura Municipal de São Luís, 1994. p.193-199.

TAKAHASHI, L. Y. Monitoramento e informatização da administração e manejo da arborização urbana. In: CONGRESSO BRASILEIRO DE ARBORIZAÇÃO URBANA, 1., 1992. Anais... Vitória, ES: PMV/SMMA, 1992. p. 119-124.

TUDINI, O. G. A arborização de acompanhamento viário e a verticalização na Zona 7 de Maringá - PR. 2006, 149f. Dissertação (Mestrado em Geografia) - Universidade Estadual de Maringá, Maringá. 2006.

VILLARINHO, F. M.; MACEDO, R. L. G.; TOMIAZZI, A. B. Avaliação da opinião pública sobre a arborização do bairro de Jacarepaguá - Freguesia, Município do Rio de Janeiro, RJ. In: CONGRESSO BRASILEIRO DE ARBORIZAÇÃO URBANA, 9., 2005. Belo Horizonte. Anais... Belo Horizonte, MG, 2005. p. 85-91. 